\title{
A Vibration Control Method for the Flexible Arm Based on Energy Migration
}

\author{
Yushu Bian, ${ }^{1}$ Zhihui Gao, ${ }^{1}$ and Ming Fan ${ }^{2}$ \\ ${ }^{1}$ School of Mechanical Engineering and Automation, Beihang University, Beijing 100191, China \\ ${ }^{2}$ The Second of Corps of Engineers, GEH PLA, Beijing 100036, China \\ Correspondence should be addressed to Yushu Bian; bian_bys@buaa.edu.cn
}

Received 19 January 2015; Revised 1 June 2015; Accepted 22 June 2015

Academic Editor: Vadim V. Silberschmidt

Copyright (c) 2015 Yushu Bian et al. This is an open access article distributed under the Creative Commons Attribution License, which permits unrestricted use, distribution, and reproduction in any medium, provided the original work is properly cited.

\begin{abstract}
A vibration control method based on energy migration is proposed to decrease vibration response of the flexible arm undergoing rigid motion. A type of vibration absorber is suggested and gives rise to the inertial coupling between the modes of the flexible arm and the absorber. By analyzing 1:2 internal resonance, it is proved that the internal resonance can be successfully created and the exchange of vibration energy is existent. Due to the inertial coupling, the damping enhancement effect is revealed. Via the inertial coupling, vibration energy of the flexible arm can be dissipated by not only the damping of the vibration absorber but also its own enhanced damping, thereby effectively decreasing vibration. Through numerical simulations and analyses, it is proven that this method is feasible in controlling nonlinear vibration of the flexible arm undergoing rigid motion.
\end{abstract}

\section{Introduction}

Although flexible robotic arms have various important applications in space exploration, automatic assembly, undersea operation, nuclear environment, and so forth, major possible disadvantages of these arms are serious vibration response and deteriorative tracking accuracy due to large dimension, light weight, low structural damping, and small stiffness. Therefore, a great deal of research has been conducted to combat these problems.

Some traditional methods have been used to control vibration, like enhancing the stiffness of links and joints, optimizing shape and dimensions $[1,2]$, and utilizing composite materials $[3,4]$. In recent years, a number of active control methods are put forward, most of them employ such smart material actuators as the piezoelectric ceramic and shape memory alloy, and remarkable progress has been made $[5,6]$. In addition to these vibration suppression methods, vibration absorption methods are especially useful to the large amplitude vibration with strong energy. However, since large amplitude can unavoidably excite nonlinear effects in the dynamic system, many methods based on linear vibration model may cause fundamental mistakes. In fact, although the nonlinearity can increase the analysis difficulty, modal interaction caused by the nonlinearity can be used to migrate and dissipate strong vibration energy of the flexible arm.

Internal resonance is a typical nonlinear principle. Through modal interaction, vibration energy of one mode can be transferred to another mode which is commensurable or nearly commensurable with the former $[7,8]$. Golnaraghi [9] firstly used internal resonance to decrease structural vibration of a flexible cantilever beam. By properly tuning the position gain, internal resonance was established between the beam and the slider. And the beam vibration was decreased by migrating and dissipating its vibration energy through the slider motion. Tuer et al. [10] proposed Disabled Torque Method (DTM) and Dissipated Energy Method (DEM) to control a flexible cantilever beam based on internal resonance. Afterwards, Oueini and Golnaraghi [11] finished an experimental study and built a controller with analog electronic components. Furthermore, Khajepour et al. [12] used center manifold theory to control vibration of the flexible beam. However, the flexible cantilever beam they have researched is a rigid beam connected by a linear torsional spring. This simplified model is not suitable for the long and thin arm which should be represented by a distributed flexibility model. 


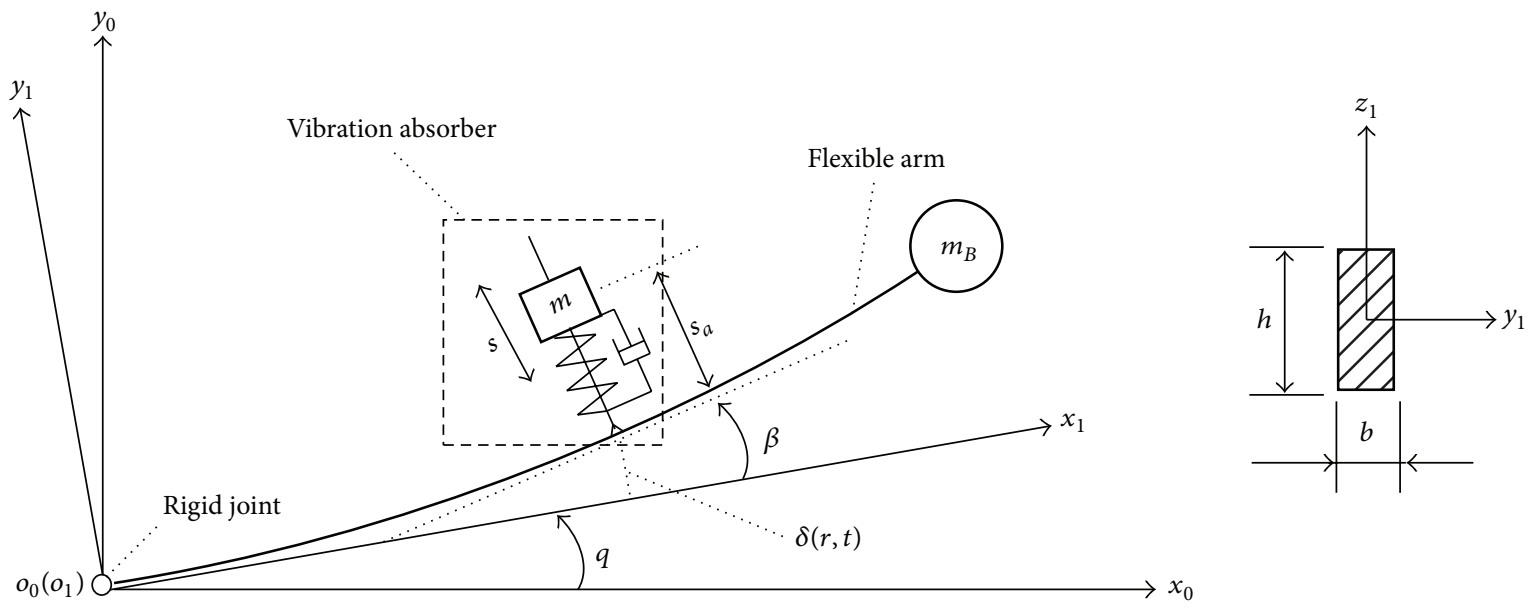

Figure 1: Model of the flexible arm with a vibration absorber.

In recent years, the above studies have been extended to control vibration of the distributed flexible beam. Pai et al. [13] used a higher order internal resonance absorber to decrease vibration of a cantilevered aluminum plate. Ashour and Nayfeh [14] designed an active vibration controller for a flexible structure based on internal resonance and saturation phenomenon. Yaman and Sen [15] studied vibration absorption problem concerning a cantilever beam with a tip mass and pendulum which is attached to the tip mass. Hui et al. [16] used the internally resonant energy transfer from the symmetrical to antisymmetrical mode to reduce the source mass vibration. But these studies only deal with the flexible cantilever beam without rigid motion, but do not involve the flexible arm undergoing large scale joint motion which exhibits much more complex dynamic behaviors.

To the best of our knowledge, there is little research on controlling vibration of the flexible arm based on energy migration. In this paper, a type of vibration absorber is suggested and gives rise to the inertial coupling between the modes of the flexible arm and the absorber. By analyzing 1:2 internal resonance, it is proved that the internal resonance can be successfully created and the exchange of vibration energy is existent. Furthermore, due to the inertial coupling, the damping enhancement effect is revealed. Via the inertial coupling, the damping of the vibration absorber can be mapped into the flexible arm, thus increasing the damping effect of the flexible arm. In this way, vibration energy of the flexible arm can not only be dissipated by the damping of the vibration absorber based on the internal resonance but also be attenuated by its own enhanced damping, thereby effectively decreasing vibration.

\section{Oscillatory Differential Equation}

In this study, a model with one flexible arm, one rigid joint, and a vibration absorber is considered, as shown in Figure 1. The arm is a uniform Euler-Bernoulli beam with the length $l$, the rectangle cross section of height $h$, and width $b$ and a tip mass $m_{B}$. Only flexural deformation $\delta\left(x_{1}, t\right)$ about $y_{1}$ axis is considered, where $t$ is time. The arm rotates around the rigid joint, and the nominal motion is denoted by $q$. The vibration absorber is a slider mass-spring-dashpot mechanism with mass $m$, stiffness $k_{2}$, and damping $c_{2}$ and attached to the flexible arm at $x_{1}=r$. The mass displacement is denoted by $s$ and equilibrium position is denoted by $s_{a}$.

Based on the assumed-modes theory, the deformation of the flexible arm can be expressed as

$$
\delta\left(x_{1}, t\right)=\sum_{i=1}^{n_{F}} u_{i}\left(x_{1}\right) \varphi_{i}(t)
$$

where $\varphi_{i}(t)$ is the $i$ th modal coordinate describing deformation of the flexible arm, $u_{i}\left(x_{1}\right)$ is the $i$ th mode shape satisfying certain geometric and force boundary conditions, and $n_{F}$ is the number of flexural degrees of freedom of the flexible arm.

In present study, only the fundamental mode of the arm is considered due to its most contribution to the vibration response in common cases. Equation (1) can be written as

$$
\delta\left(x_{1}, t\right)=u_{1}\left(x_{1}\right) \varphi_{1}(t)
$$

The angle of the tangent of the flexible arm at $x_{1}=r$ with respect to $x_{1}$ axis is denoted by $\beta$, as shown in Figure 1 . Consider

$$
\beta=\tilde{\beta}_{r} \varphi_{1}(t)
$$

where $\widetilde{\beta}_{r}=\left.\left(d u_{1}\left(x_{1}\right) / d x_{1}\right)\right|_{x_{1}=r}$. The axial displacement caused by the transverse bending of the arm is written as

$$
\kappa\left(x_{1}\right)=-\frac{1}{2} \int_{0}^{x_{1}}\left(\frac{\partial \delta(\vartheta, t)}{\partial \vartheta}\right)^{2} d \vartheta=-\frac{1}{2} B_{1} \varphi_{1}^{2},
$$

where $\vartheta$ is a dummy variable and $B_{1}=\int_{0}^{x_{1}}\left(d u_{1} / d \vartheta\right)^{2} d \vartheta$.

To use the absorber for reducing vibration response that resulted from the fundamental mode of the arm, the coupling effect between the fundamental mode coordinate $\varphi_{1}$ of the arm and the degree of freedom $s$ of the absorber is our 
concern. Based on Kane's method, the dynamic equations concerning $\varphi_{1}$ and $s$ are derived and can be written as

$$
\begin{aligned}
m_{11} \ddot{\varphi}_{1} & +m_{12} \ddot{s}+c_{11} \dot{\varphi}_{1}+\left(k_{11}+b_{1} \ddot{q}\right) \varphi_{1}+k_{12} \ddot{q} s \\
= & -b_{2} \dot{\varphi}_{1} \dot{s}-b_{3} \dot{\varphi}_{1}^{2}-b_{4} \ddot{\varphi}_{1} \varphi_{1}-b_{2} \ddot{\varphi}_{1} s-b_{5} \ddot{q}-b_{6} \dot{q}^{2} \\
& \quad+f_{1}\left(\varphi_{1}, \dot{\varphi}_{1}, \ddot{\varphi}_{1}, s, \dot{s}, \ddot{s}, \dot{q}, \ddot{q}\right), \\
m_{22} \ddot{s}+ & m_{21} \ddot{\varphi}_{1}+c_{22} \dot{s}+k_{22} s \\
= & b_{7} \dot{\varphi}_{1}^{2}-b_{8} \ddot{q}+b_{9} \dot{q}^{2}+f_{2}\left(\varphi_{1}, \dot{\varphi}_{1}, \ddot{\varphi}_{1}, s, \dot{s}, \ddot{s}, \dot{q}, \ddot{q}\right),
\end{aligned}
$$

where $m_{11}=B_{2}+m_{B} u_{1 l}^{2}+m s_{a}^{2} \widetilde{\beta}_{r}^{2}+m u_{1 r}^{2} ; B_{2}=\rho \int_{0}^{l} u_{1}^{2}\left(x_{1}\right) d x_{1}$; $u_{1 l}=\left.u_{1}\left(x_{1}\right)\right|_{x_{1}=l} ; u_{1 r}=\left.u_{1}\left(x_{1}\right)\right|_{x_{1}=r} ; m_{12}=m u_{1 r} ; c_{11}$ is the damping of the arm; $k_{11}=E I \int_{0}^{l}\left(d^{4} u_{1}\left(x_{1}\right) / d x_{1}{ }^{4}\right) u_{1}\left(x_{1}\right) d x_{1}$; $b_{1}=m s_{a}\left(B_{1 r}-r \widetilde{\beta}_{r}^{2}\right) ; B_{1 r}=\left.B_{1}\left(x_{1}\right)\right|_{x_{1}=r} ; k_{12}=2 m s_{a} \widetilde{\beta}_{r} ;$ $b_{2}=2 m s_{a} \widetilde{\beta}_{r}^{2} ; b_{3}=m s_{a} \widetilde{\beta}_{r}\left(B_{1 r}-u_{1 r} \widetilde{\beta}_{r}\right) ; b_{4}=2 b_{3} ; b_{5}=$ $\rho \int_{0}^{l} x u_{1}\left(x_{1}\right) d x_{1}+m_{B} l u_{1 l}+m s_{a}^{2} \widetilde{\beta}_{r}+m r u_{1 r} ; b_{6}=m s_{a}\left(r \widetilde{\beta}_{r}-u_{1 r}\right)$; $m_{22}=m ; c_{22}=c_{2} ; k_{22}=k_{2} ; m_{21}=m_{12} ; b_{7}=m s_{a} \widetilde{\beta}_{r}^{2} ; b_{8}=m r ;$ $b_{9}=m s_{a} ; \rho$ is mass per length of the flexible arm; $E I$ is flexural rigidity; $l$ is the length of the arm.

\section{Nonlinear Analysis}

Equations (5) are nonlinear differential equations and are solved in this section.

3.1. Nondimensionalization and Decoupling of the Equations of Motion. The nondimensional variables $\varphi^{*}, s^{*}, \tau$, and $q^{*}$ are defined by

$$
\begin{aligned}
\varphi^{*} & =\frac{\varphi_{1}}{l}, \\
s^{*} & =\frac{s}{l}, \\
\tau & =\omega_{\varphi} t, \\
q^{*} & =q,
\end{aligned}
$$

where $\omega_{\varphi}=\sqrt{k_{11} / m_{11}}$ and the equations of motion (5) are nondimensionalized as follows:

$$
\begin{aligned}
& \frac{d^{2} \varphi^{*}}{d \tau^{2}}+\widetilde{m}_{12} \frac{d^{2} s^{*}}{d \tau^{2}}+\eta_{11} \frac{d \varphi^{*}}{d \tau}+\left(1+d_{1} \frac{d^{2} q^{*}}{d \tau^{2}}\right) \varphi^{*} \\
& +\widetilde{k}_{12} \frac{d^{2} q^{*}}{d \tau^{2}} s^{*}=-d_{2} \frac{d \varphi^{*}}{d \tau} \frac{d s^{*}}{d \tau}-d_{3}\left(\frac{d \varphi^{*}}{d \tau}\right)^{2} \\
& -d_{4} \frac{d^{2} \varphi^{*}}{d \tau^{2}} \varphi^{*}-d_{2} \frac{d^{2} \varphi^{*}}{d \tau^{2}} s^{*}-d_{5} \frac{d^{2} q^{*}}{d \tau^{2}}-d_{6}\left(\frac{d q^{*}}{d \tau}\right)^{2} \\
& +f_{3}\left(\varphi^{*}, \frac{d \varphi^{*}}{d \tau}, \frac{d^{2} \varphi^{*}}{d \tau^{2}}, s^{*}, \frac{d s^{*}}{d \tau}, \frac{d^{2} s^{*}}{d \tau^{2}}, \frac{d q^{*}}{d \tau}, \frac{d^{2} q^{*}}{d \tau^{2}}\right),
\end{aligned}
$$

$$
\begin{aligned}
& \frac{d^{2} s^{*}}{d \tau^{2}}+\widetilde{m}_{21} \frac{d^{2} \varphi^{*}}{d \tau^{2}}+\eta_{22} \frac{d s^{*}}{d \tau}+\omega_{s \varphi}^{2} s^{*}=d_{7}\left(\frac{d \varphi^{*}}{d \tau}\right)^{2} \\
& -d_{8} \frac{d^{2} q^{*}}{d \tau^{2}}+d_{9}\left(\frac{d q^{*}}{d \tau}\right)^{2} \\
& +f_{4}\left(\varphi^{*}, \frac{d \varphi^{*}}{d \tau}, \frac{d^{2} \varphi^{*}}{d \tau^{2}}, s^{*}, \frac{d s^{*}}{d \tau}, \frac{d^{2} s^{*}}{d \tau^{2}}, \frac{d q^{*}}{d \tau}, \frac{d^{2} q^{*}}{d \tau^{2}}\right),
\end{aligned}
$$

where $\widetilde{m}_{12}=m_{12} / m_{11} ; \eta_{11}=c_{11} /\left(\omega_{\varphi} m_{11}\right) ; d_{1}=b_{1} / m_{11} ; \widetilde{k}_{12}=$ $k_{12} / m_{11} ; d_{2}=b_{2} l / m_{11} ; d_{3}=b_{3} l / m_{11} ; d_{4}=b_{4} l / m_{11} ; d_{5}=$ $b_{5} /\left(m_{11} l\right) ; d_{6}=b_{6} /\left(m_{11} l\right) ; \widetilde{m}_{21}=m_{21} / m_{22} ; \eta_{22}=c_{22} /\left(\omega_{\varphi} m_{22}\right)$; $\omega_{s}=\sqrt{k_{22} / m_{22}} ; \omega_{s \varphi}=\omega_{s} / \omega_{\varphi} ; d_{7}=b_{7} l / m_{22} ; d_{8}=b_{8} /\left(m_{22} l\right)$; $d_{9}=b_{9} /\left(m_{22} l\right)$.

To make the damping and nonlinearities appear in the same perturbation equations, let $\eta_{11}=\varepsilon \zeta_{11}, \eta_{22}=\varepsilon \zeta_{22}$, $\varphi^{*}=\varepsilon \phi, s^{*}=\varepsilon w$, and $d q^{*} / d \tau=\varepsilon(d \chi / d \tau)$, where $\varepsilon$ is a small nondimensional bookkeeping parameter, $0<\varepsilon \ll 1$. Then, (7) can be expressed as

$$
\begin{aligned}
& \frac{d^{2} \phi}{d \tau^{2}}+\phi=-\widetilde{m}_{12} \frac{d^{2} w}{d \tau^{2}}-d_{5} \frac{d^{2} \chi}{d \tau^{2}}+\varepsilon\left[-\zeta_{11} \frac{d \phi}{d \tau}\right. \\
& -d_{1} \frac{d^{2} \chi}{d \tau^{2}} \phi-\widetilde{k}_{12} \frac{d^{2} \chi}{d \tau^{2}} w-d_{2} \frac{d \phi}{d \tau} \frac{d w}{d \tau}-d_{3}\left(\frac{d \phi}{d \tau}\right)^{2} \\
& \left.-d_{4} \frac{d^{2} \phi}{d \tau^{2}} \phi-d_{2} \frac{d^{2} \phi}{d \tau^{2}} w-d_{6}\left(\frac{d \chi}{d \tau}\right)^{2}\right]+o(\varepsilon), \\
& \frac{d^{2} w}{d \tau^{2}}+\omega_{s \varphi}^{2} w=-\widetilde{m}_{21} \frac{d^{2} \phi}{d \tau^{2}}-d_{8} \frac{d^{2} \chi}{d \tau^{2}}+\varepsilon\left[-\zeta_{22} \frac{d w}{d \tau}\right. \\
& \left.+d_{7}\left(\frac{d \phi}{d \tau}\right)^{2}+d_{9}\left(\frac{d \chi}{d \tau}\right)^{2}\right]+o(\varepsilon) .
\end{aligned}
$$

3.2. Perturbation Analysis. The time dependence $\tau$ is expanded in terms of multiple time scales, $T_{i}=\varepsilon^{i} \tau,(i=$ $0,1, \ldots)$, so that the first- and second-time derivatives become

$$
\begin{aligned}
\frac{d}{d \tau} & =D_{0}+\varepsilon D_{1}+\varepsilon^{2} D_{2}+\cdots, \\
\frac{d^{2}}{d \tau^{2}} & =D_{0}^{2}+2 \varepsilon D_{0} D_{1}+\varepsilon^{2} D_{1}+2 \varepsilon^{2} D_{0} D_{2}+\cdots,
\end{aligned}
$$

where $D_{i}=\partial / \partial T_{i},(i=0,1, \ldots)$.

We seek first-order approximate solutions of (8) and (9) by using the method of multiple scales in the form

$$
\begin{aligned}
& \phi(\tau, \varepsilon)=\phi_{0}\left(T_{0}, T_{1}\right)+\varepsilon \phi_{1}\left(T_{0}, T_{1}\right), \\
& w(\tau, \varepsilon)=w_{0}\left(T_{0}, T_{1}\right)+\varepsilon w_{1}\left(T_{0}, T_{1}\right) .
\end{aligned}
$$

Substituting (10), (11) into (8) and (9) and equating coefficients of same powers of $\varepsilon$, we obtain the following:

Order $\left(\varepsilon^{0}\right)$ is as follows:

$$
\begin{aligned}
D_{0}^{2} \phi_{0}+\phi_{0} & =-\widetilde{m}_{12} D_{0}^{2} w_{0}+g_{1}, \\
D_{0}^{2} w_{0}+\omega_{s \varphi}^{2} w_{0} & =-\widetilde{m}_{21} D_{0}^{2} \phi_{0}+g_{2} .
\end{aligned}
$$


Order $\left(\varepsilon^{1}\right)$ is as follows:

$$
\begin{aligned}
D_{0}^{2} \phi_{1}+\phi_{1}= & -2 D_{0} D_{1} \phi_{0}-\zeta_{11} D_{0} \phi_{0}-\widetilde{m}_{12} D_{0}^{2} w_{1} \\
& -2 \widetilde{m}_{12} D_{0} D_{1} w_{0}-d_{1} D_{0}^{2} \chi \phi_{0} \\
& -\widetilde{k}_{12} D_{0}^{2} \chi w_{0}-d_{2} D_{0} \phi_{0} D_{0} w_{0} \\
& -d_{3}\left(D_{0} \phi_{0}\right)^{2}-d_{4} D_{0}^{2} \phi_{0} \phi_{0} \\
& -d_{2} D_{0}^{2} \phi_{0} w_{0}-d_{6}\left(D_{0} \chi\right)^{2} \\
D_{0}^{2} w_{1}+\omega_{s \varphi}^{2} w_{1}= & -2 D_{0} D_{1} w_{0}-\widetilde{m}_{21} D_{0}^{2} \varphi_{1} \\
& -2 \widetilde{m}_{21} D_{0} D_{1} \phi_{0}-\zeta_{22} D_{0} w_{0} \\
& +d_{7}\left(D_{0} \phi_{0}\right)^{2}+d_{9}\left(D_{0} \chi\right)^{2}
\end{aligned}
$$

where $g_{1}=-d_{5} D_{0}^{2} \chi, g_{2}=-d_{8} D_{0}^{2} \chi$.

The solution of (12) can be written in the form

$$
\begin{aligned}
\phi_{0}= & A_{1}\left(T_{1}\right) \exp \left(j \omega_{1} T_{0}\right)+A_{2}\left(T_{1}\right) \exp \left(j \omega_{2} T_{0}\right) \\
& +h_{1}+c c, \\
w_{0}= & \Lambda_{1} A_{1}\left(T_{1}\right) \exp \left(j \omega_{1} T_{0}\right) \\
& +\Lambda_{2} A_{2}\left(T_{1}\right) \exp \left(j \omega_{2} T_{0}\right)+h_{2}+c c,
\end{aligned}
$$

where $A_{1}\left(T_{1}\right)$ and $A_{2}\left(T_{1}\right)$ are functions of slow time $T_{1}$; $h_{1}=g_{1} / 2 ; h_{2}=g_{2} /\left(2 \omega_{s \varphi}^{2}\right) ; c c$ denotes the complex conjugate terms. According to the theory of ordinary differential equation, the $\omega_{n}^{2}(n=1,2)$ are the roots of

$$
\begin{aligned}
& \left(1-\widetilde{m}_{12} \widetilde{m}_{21}\right) \omega_{n}^{4}-\left(1+\omega_{s \varphi}^{2}\right) \omega_{n}^{2}+\omega_{s \varphi}^{2}=0, \\
& \Lambda_{n}=\frac{1-\omega_{n}^{2}}{\omega_{n}^{2} \widetilde{m}_{12}}=\frac{\widetilde{m}_{21} \omega_{n}^{2}}{\omega_{s \varphi}^{2}-\omega_{n}^{2}}
\end{aligned}
$$

and $\omega_{n}$ are assumed to be distinct.

In this study, because the second-order nonlinear coupling terms exist in the dynamic model, the vibration absorber is used to control vibration of the flexible arm at the $1: 2$ internal resonance condition; that is, $\omega_{2} \approx 2 \omega_{1}$. It is this internal resonance condition that enables the transfer of vibration energy between the fundamental mode of the arm and the vibration mode of the absorber. In order to solve the nonlinear problem, substituting (14) into (13) yields

$$
\begin{aligned}
& D_{0}^{2} \phi_{1}+\phi_{1}=-\left(2 j \omega_{1} A_{1}^{\prime}+\zeta_{11} j \omega_{1} A_{1}+2 \widetilde{m}_{12} j \omega_{1} \Lambda_{1} A_{1}^{\prime}\right. \\
& \left.\quad+d_{1} D_{0}^{2} \chi A_{1}+\widetilde{k}_{12} D_{0}^{2} \chi \Lambda_{1} A_{1}\right) \exp \left(j \omega_{1} T_{0}\right) \\
& \quad+\left[-d_{2}\left(\Lambda_{1}+\Lambda_{2}\right) \omega_{1} \omega_{2}-2 d_{3} \omega_{1} \omega_{2}\right. \\
& \left.\quad+d_{4}\left(\omega_{1}^{2}+\omega_{2}^{2}\right)+d_{2}\left(\Lambda_{2} \omega_{1}^{2}+\Lambda_{1} \omega_{2}^{2}\right)\right] \bar{A}_{1} A_{2} \\
& \quad \cdot \exp \left[j\left(\omega_{2}-\omega_{1}\right) T_{0}\right]+\mathrm{NST}+c c,
\end{aligned}
$$

$$
\begin{aligned}
& D_{0}^{2} w_{1}+\omega_{s \varphi}^{2} w_{1}=-\left(2 j \omega_{2} \Lambda_{2} A_{2}^{\prime}+\zeta_{22} j \omega_{2} \Lambda_{2} A_{2}\right. \\
& \left.+2 \widetilde{m}_{21} j \omega_{2} A_{2}^{\prime}\right) \exp \left(j \omega_{2} T_{0}\right)-d_{7} \omega_{1}^{2} A_{1}^{2} \exp \left(2 j \omega_{1} T_{0}\right) \\
& \quad+\mathrm{NST}+c c,
\end{aligned}
$$

where ()$^{\prime} \equiv \partial() / \partial T_{1}$ and NST denotes nonsecular terms.

In the case of the $1: 2$ internal resonance, a detuning parameter $\sigma$ is introduced as $\omega_{2}=2 \omega_{1}+\varepsilon \sigma$. To determine the solvability conditions of (17), the particular solutions are sought in the form

$$
\begin{aligned}
& \phi_{1}=p_{11} \exp \left(j \omega_{1} T_{0}\right)+p_{12} \exp \left(j \omega_{2} T_{0}\right), \\
& w_{1}=p_{21} \exp \left(j \omega_{1} T_{0}\right)+p_{22} \exp \left(j \omega_{2} T_{0}\right) .
\end{aligned}
$$

Substituting (18) into (17) and then equating the coefficients of $\exp \left(j \omega_{1} T_{0}\right)$ and $\exp \left(j \omega_{2} T_{0}\right)$ on both sides, one obtains

$$
\begin{aligned}
\left(1-\omega_{n}^{2}\right) p_{1 n}-\widetilde{m}_{12} \omega_{n}^{2} p_{2 n} & =R_{1 n}, \\
-\widetilde{m}_{21} \omega_{n}^{2} p_{1 n}+\left(\omega_{s \varphi}^{2}-\omega_{n}^{2}\right) p_{2 n} & =R_{2 n},
\end{aligned}
$$

where

$$
\begin{aligned}
& R_{11}=-2 j \omega_{1}\left(1+\widetilde{m}_{12} \Lambda_{1}\right) A_{1}^{\prime}-j \omega_{1} \zeta_{11} A_{1} \\
& +\left(-d_{1} D_{0}^{2} \chi-\widetilde{k}_{12} D_{0}^{2} \chi \Lambda_{1}+2 h_{1} d_{4} \omega_{1}^{2}+2 h_{2} d_{2} \omega_{1}^{2}\right) \\
& \cdot A_{1}+\left[d_{4}\left(\omega_{1}^{2}+\omega_{2}^{2}\right)-d_{2}\left(\Lambda_{1}+\Lambda_{2}\right) \omega_{1} \omega_{2}\right. \\
& \left.\quad-2 d_{3} \omega_{1} \omega_{2}+d_{2}\left(\Lambda_{2} \omega_{1}^{2}+\Lambda_{1} \omega_{2}^{2}\right)\right] \bar{A}_{1} A_{2} \exp \left(j \sigma T_{1}\right), \\
& R_{12}=-2 j \omega_{2}\left(1+\widetilde{m}_{12} \Lambda_{2}\right) A_{2}^{\prime}-j \omega_{2} \zeta_{11} A_{2} \\
& +\left(-d_{1} D_{0}^{2} \chi-\widetilde{k}_{12} D_{0}^{2} \chi \Lambda_{2}+2 h_{1} d_{4} \omega_{2}^{2}+2 h_{2} d_{2} \omega_{2}^{2}\right) \\
& \cdot A_{2}+\left(2 d_{2} \Lambda_{1}+d_{3}+d_{4}\right) \omega_{1}^{2} A_{1}^{2} \exp \left(-j \sigma T_{1}\right), \\
& R_{21}=-2 j \omega_{1}\left(\widetilde{m}_{21}+\Lambda_{1}\right) A_{1}^{\prime}-j \omega_{1} \zeta_{22} \Lambda_{1} A_{1} \\
& +2 d_{7} \omega_{1} \omega_{2} \bar{A}_{1} A_{2} \exp \left(j \sigma T_{1}\right), \\
& R_{22}=-2 j \omega_{2}\left(\widetilde{m}_{21}+\Lambda_{2}\right) A_{2}^{\prime}-j \omega_{2} \zeta_{22} \Lambda_{2} A_{2} \\
& -d_{7} \omega_{1}^{2} A_{1}^{2} \exp \left(-j \sigma T_{1}\right) .
\end{aligned}
$$

Therefore, the problem of determining the solvability conditions of (17) is reduced to that of determining the solvability condition of (19).

Since the determinant of the coefficient matrix of (19) is zero according to (15), the solvability conditions are

$$
\begin{aligned}
\left|\begin{array}{cc}
R_{1 n} & -\widetilde{m}_{12} \omega_{n}^{2} \\
R_{2 n} & \omega_{s \phi}^{2}-\omega_{n}^{2}
\end{array}\right| & =0 \\
\text { or } R_{1 n} & =-\Lambda_{n} R_{2 n} \frac{\widetilde{m}_{12}}{\widetilde{m}_{21}}
\end{aligned}
$$

on account of (16) 
Substituting (20) into (21) yields

$$
\begin{aligned}
- & 2 j \omega_{1}\left(1+\widetilde{m}_{12} \Lambda_{1}\right) A_{1}^{\prime}-j \omega_{1} \zeta_{11} A_{1}+\left(-d_{1} D_{0}^{2} \chi\right. \\
- & \left.\widetilde{k}_{12} D_{0}^{2} \chi \Lambda_{1}+2 h_{1} d_{4} \omega_{1}^{2}+2 h_{2} d_{2} \omega_{1}^{2}\right) A_{1} \\
+ & {\left[d_{4}\left(\omega_{1}^{2}+\omega_{2}^{2}\right)-d_{2}\left(\Lambda_{1}+\Lambda_{2}\right) \omega_{1} \omega_{2}-2 d_{3} \omega_{1} \omega_{2}\right.} \\
& \left.+d_{2}\left(\Lambda_{2} \omega_{1}^{2}+\Lambda_{1} \omega_{2}^{2}\right)\right] \bar{A}_{1} A_{2} \exp \left(j \sigma T_{1}\right)=-\Lambda_{1} \\
& \cdot \frac{\widetilde{m}_{12}}{\widetilde{m}_{21}}\left[-2 j \omega_{1}\left(\widetilde{m}_{21}+\Lambda_{1}\right) A_{1}^{\prime}\right. \\
& \left.-j \omega_{1} \zeta_{22} \Lambda_{1} A_{1}+2 d_{7} \omega_{1} \omega_{2} \bar{A}_{1} A_{2} \exp \left(j \sigma T_{1}\right)\right], \\
- & 2 j \omega_{2}\left(1+\widetilde{m}_{12} \Lambda_{2}\right) A_{2}^{\prime}-j \omega_{2} \zeta_{11} A_{2}+\left(-d_{1} D_{0}^{2} \chi\right. \\
& \left.-\widetilde{k}_{12} D_{0}^{2} \chi \Lambda_{2}+2 h_{1} d_{4} \omega_{2}^{2}+2 h_{2} d_{2} \omega_{2}^{2}\right) A_{2}+\left(2 d_{2} \Lambda_{1}\right. \\
& \left.+d_{3}+d_{4}\right) \omega_{1}^{2} A_{1}^{2} \exp \left(-j \sigma T_{1}\right)=-\Lambda_{2} \\
& +\widetilde{m}_{12}\left[-2 j \omega_{2}\left(\widetilde{m}_{21}+\Lambda_{2}\right) A_{2}^{\prime}\right. \\
& \widetilde{m}_{21} \\
- & \left.j \omega_{2} \zeta_{22} \Lambda_{2} A_{2}-d_{7} \omega_{1}^{2} A_{1}^{2} \exp \left(-j \sigma T_{1}\right)\right] .
\end{aligned}
$$

It is convenient to express the resulting modulation equations in polar form by introducing the following transformation:

$$
\begin{aligned}
& A_{1}=\frac{1}{2} a_{1} \exp \left(j \theta_{1}\right), \\
& A_{2}=\frac{1}{2} a_{2} \exp \left(j \theta_{2}\right),
\end{aligned}
$$

where $a_{1}, a_{2}, \theta_{1}$, and $\theta_{2}$ are real functions of the slow time $T_{1}$; $a_{1}$ and $a_{2}$ are defined as the modal amplitudes.

Inserting (23) into (22), then setting the coefficients of the real and imaginary parts to zero yields the modulation equations

$$
\begin{aligned}
a_{1}^{\prime} & =-\frac{h_{2}}{2 h_{1}} a_{1}+\frac{h_{3}}{4 h_{1}} a_{1} a_{2} \sin \gamma, \\
a_{2}^{\prime} & =-\frac{h_{5}}{2 h_{4}} a_{2}-\frac{h_{6}}{4 h_{4}} a_{1}^{2} \sin \gamma, \\
\theta_{1}^{\prime} & =-\frac{h_{7}}{2 h_{1}}-\frac{h_{3}}{4 h_{1}} a_{2} \cos \gamma, \\
a_{2} \theta_{2}^{\prime} & =-\frac{h_{8}}{2 h_{4}} a_{2}-\frac{h_{6}}{4 h_{4}} a_{1}^{2} \cos \gamma, \\
\gamma & =\theta_{2}-2 \theta_{1}+\sigma T_{1}
\end{aligned}
$$

where

$$
\begin{aligned}
& h_{1}=\left(1+2 \widetilde{m}_{12} \Lambda_{1}+\frac{\Lambda_{1}^{2} \widetilde{m}_{12}}{\widetilde{m}_{21}}\right) \omega_{1} \\
& h_{2}=\left(\zeta_{11}+\frac{\Lambda_{1}^{2} \zeta_{22} \widetilde{m}_{12}}{\widetilde{m}_{21}}\right) \omega_{1} ;
\end{aligned}
$$

$$
\begin{aligned}
h_{3}= & d_{4}\left(\omega_{1}^{2}+\omega_{2}^{2}\right)-d_{2}\left(\Lambda_{1}+\Lambda_{2}\right) \omega_{1} \omega_{2}-2 d_{3} \omega_{1} \omega_{2} \\
& +d_{2}\left(\Lambda_{2} \omega_{1}^{2}+\Lambda_{1} \omega_{2}^{2}\right)+\frac{2 \Lambda_{1} d_{7} \omega_{1} \omega_{2} \widetilde{m}_{12}}{\widetilde{m}_{21}} ; \\
h_{4}= & \left(1+2 \widetilde{m}_{12} \Lambda_{2}+\frac{\Lambda_{2}^{2} \widetilde{m}_{12}}{\widetilde{m}_{21}}\right) \omega_{2} ; \\
h_{5}= & \left(\zeta_{11}+\frac{\Lambda_{2}^{2} \zeta_{22} \widetilde{m}_{12}}{\widetilde{m}_{21}}\right) \omega_{2} ; \\
h_{6}= & \left(2 d_{2} \Lambda_{1}+d_{3}+d_{4}\right) \omega_{1}^{2}-\frac{\Lambda_{2} d_{7} \omega_{1}^{2} \widetilde{m}_{12}}{\widetilde{m}_{21}} ; \\
h_{7}= & -d_{1} D_{0}^{2} \chi-\widetilde{k}_{12} D_{0}^{2} \chi \Lambda_{1}+2 h_{1} d_{4} \omega_{1}^{2}+2 h_{2} d_{2} \omega_{1}^{2} ; \\
h_{8}= & -d_{1} D_{0}^{2} \chi-\widetilde{k}_{12} D_{0}^{2} \chi \Lambda_{2}+2 h_{1} d_{4} \omega_{2}^{2}+2 h_{2} d_{2} \omega_{2}^{2} .
\end{aligned}
$$

Eliminating $\theta_{1}$ and $\theta_{2}$ from (26) and (27) yields

$$
\begin{aligned}
a_{2} \gamma^{\prime}= & \sigma a_{2}-\frac{h_{8}}{2 h_{4}} a_{2}-\frac{h_{6}}{4 h_{4}} a_{1}^{2} \cos \gamma+\frac{h_{7}}{h_{1}} a_{2} \\
& +\frac{h_{3}}{2 h_{1}} a_{2}^{2} \cos \gamma .
\end{aligned}
$$

From $h_{2}$ and $h_{5}$, it can be seen that both $\zeta_{11}$ and $\zeta_{22}$ affect $a_{1}$ and $a_{2}$ and thus affect the response of both flexible arm and the vibration absorber. In this way, vibration energy of the flexible manipulator can not only be dissipated by the damping of the vibration absorber based on the internal resonance but also be attenuated by its own enhanced damping, thereby obtaining better vibration control results. In this paper, it is called "the damping enhancement effect."

3.3. Analysis of Secular Term. In order to better understand the dynamics of the system, the undamped case (i.e., $\zeta_{11}=$ $\left.\zeta_{22}=0\right)$ is studied. We have

$$
\begin{aligned}
& a_{1}^{\prime}=\frac{h_{3}}{4 h_{1}} a_{1} a_{2} \sin \gamma, \\
& a_{2}^{\prime}=-\frac{h_{6}}{4 h_{4}} a_{1}^{2} \sin \gamma .
\end{aligned}
$$

Let

$$
v=\frac{h_{1} h_{6}}{h_{3} h_{4}} .
$$

Then multiplying (31) by $a_{1}$ and (32) by $v a_{2}$ and adding them, it results in the equation

$$
a_{1} a_{1}^{\prime}+v a_{2} a_{2}^{\prime}=0
$$

Integrating (34), one obtains

$$
a_{1}^{2}+v a_{2}^{2}=E_{0}=\text { const., }
$$


where $E_{0}$ is a constant representing the total energy and is dependent on initial conditions.

From (33), it is seen that $v$ is determined by the structural parameters of both the flexible arm and the vibration absorber. If the structural parameters of the flexible arm are given, then $v$ will be uniquely determined by those of the vibration absorber, that is, $m, s_{a}$, and $r$. Due to the difficulty in determining the sign of $v$ in symbolic form, a numerical method is used here. Firstly, $m / m_{l}, s_{a} / l$, and $r / l$ are viewed as three search variables, where $m_{l}$ is the mass of the flexible arm. Secondly, the search ranges of these variables are defined, respectively. In this example, $m / m_{l} \in(0,0.5]$, $s_{a} / l \in(0,0.2]$, and $r / l \in(0,1]$. Thirdly, the search step is given. In this example, the search step is 0.001 . Then, a numerical method is used to find the sign of $v$ in terms of the given search variables, search ranges, and search step. The final search result is $v>0$ when $m / m_{l} \in(0,0.5]$, $s_{a} / l \in(0,0.2]$, and $r / l \in(0,1]$. As a result, $a_{1}$ and $a_{2}$ in (35) are always bounded. Since the damping is neglected in this case, the system is conservative and the energy level remains constant. Therefore, if the response of the modal amplitude $a_{1}$ is periodic, $a_{2}$ will be periodic and out of phase from $a_{1}$. Equation (35) demonstrates that, in the absence of damping, the energy in the system continues to be exchanged undamped between the fundamental mode of the flexible arm and the vibration mode of the absorber.

3.4. The Dissipative Energy Method. In the presence of damping (i.e., $\zeta_{11}>0$ and $\zeta_{22}>0$ ), the equilibrium points for this case are defined by the solution of the set of steady state secular term equations; namely,

$$
\begin{aligned}
& -\frac{h_{2}}{2 h_{1}} a_{1}+\frac{h_{3}}{4 h_{1}} a_{1} a_{2} \sin \gamma=0, \\
& -\frac{h_{5}}{2 h_{4}} a_{2}-\frac{h_{6}}{4 h_{4}} a_{1}^{2} \sin \gamma=0, \\
& \sigma a_{2}-\frac{h_{8}}{2 h_{4}} a_{2}-\frac{h_{6}}{4 h_{4}} a_{1}^{2} \cos \gamma+\frac{h_{7}}{h_{1}} a_{2}+\frac{h_{3}}{2 h_{1}} a_{2}^{2} \cos \gamma \\
& =0 .
\end{aligned}
$$

By inspection, it is determined that the system possesses an infinite number of equilibrium points defined by

$$
\begin{gathered}
a_{1}=0, \\
a_{2}=0, \\
\gamma \in R .
\end{gathered}
$$

Therefore, by examining the Jacobian, we can ascertain the stability of the system.

The Jacobian matrix of this case is

$$
\left[\begin{array}{ccc}
\mu_{1} & 0 & 0 \\
0 & \mu_{2} & 0 \\
0 & 0 & 0
\end{array}\right],
$$

where $\mu_{1}=-h_{2} / 2 h_{1}, \mu_{2}=-h_{5} / 2 h_{4}$.

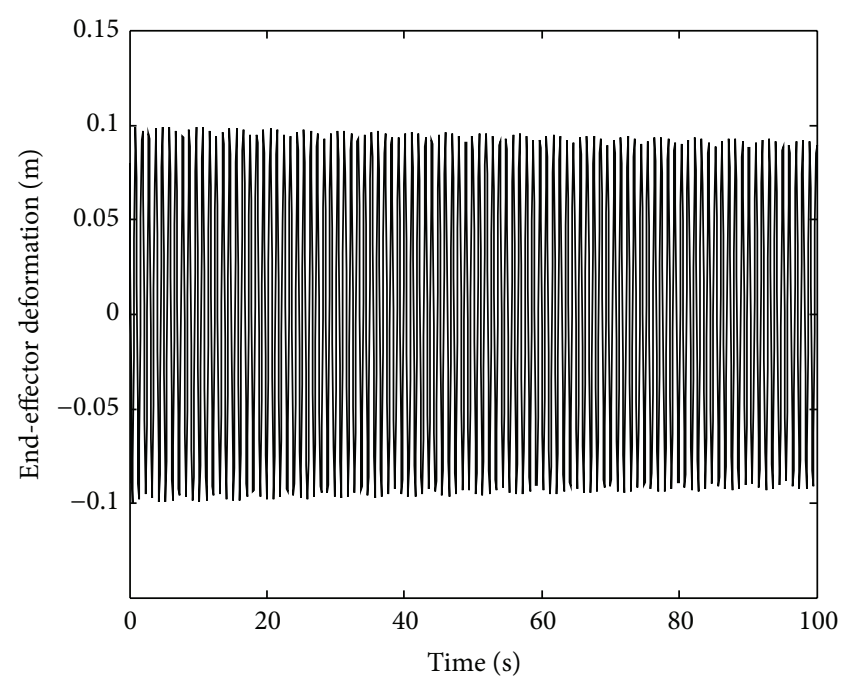

FIGURE 2: Response of the uncontrolled fixed flexible arm.

The corresponding eigenvalues are $\left(\mu_{1}, \mu_{2}, 0\right)$. Since $h_{2}>$ 0 , the sign of $\mu_{1}$ is determined by $h_{1}$. Since $h_{5}>0$, the sign of $\mu_{2}$ is determined by $h_{4}$. Similarly, due to the difficulty in determining the sign of $h_{1}$ and $h_{4}$ in symbolic form, a numerical method is used. Since $h_{1}$ and $h_{4}$ have relation to $m, s_{a}$, and $r$ when the structural parameters of the flexible arm are given, $\mathrm{m} / \mathrm{m}_{\mathrm{l}}, \mathrm{s}_{a} / \mathrm{l}$, and $r / \mathrm{l}$ are viewed as three search variables and $m / m_{l} \in(0,0.5], s_{a} / l \in(0,0.2]$, and $r / l \in(0,1]$ are defined, respectively, in this example. Also, the search step is 0.001 . The search result is $\mu_{1}<0$ and $\mu_{2}<0$ when $m / m_{l} \in(0,0.5], s_{a} / l \in$ $(0,0.2]$, and $r / l \in(0,1]$. Therefore, the modal amplitudes $a_{1}$ and $a_{2}$ are stable, as indicated by the negative eigenvalues.

By numerical integrations of (24)-(28), vibration energy is transferred between the fundamental mode of the flexible arm and the vibration mode of the absorber in the presence of damping, but it is gradually dissipated. Therefore, internal resonance can be used to reduce vibration of the flexible arm.

\section{Simulations and Analyses}

To verify the above theoretical analysis, some numerical simulations are done on the conditions: $l=1.0 \mathrm{~m}, h=0.05 \mathrm{~m}$, $b=0.003 \mathrm{~m}, m_{B}=0.5 \mathrm{~kg}$, the arm is made of aluminum, and $\rho=2710 \mathrm{~kg} / \mathrm{m}^{3}$.

Supposing the flexible arm has neither rigid motion (i.e., $q=\dot{q}=\ddot{q}=0$ ) nor vibration absorber, it can be viewed as a flexible structure. For the initial disturbance of $\delta(l, 0)=$ $0.1 \mathrm{~m}$, the end-effector deformation is obtained using a fourth-order Runge-Kutta numerical integration algorithm, as shown in Figure 2. For a flexible structure subjected to an initial disturbance, its end-effector response is usually a slowly attenuated vibration due to the low damping. To verify the above results, a virtual prototype simulation is conducted using ADAMS software. The dynamic model of the flexible arm with the same parameters is created (as shown in Figure 3), and the end-effector deformation is shown in Figure 4. As can be seen, the results are similar to each other. 


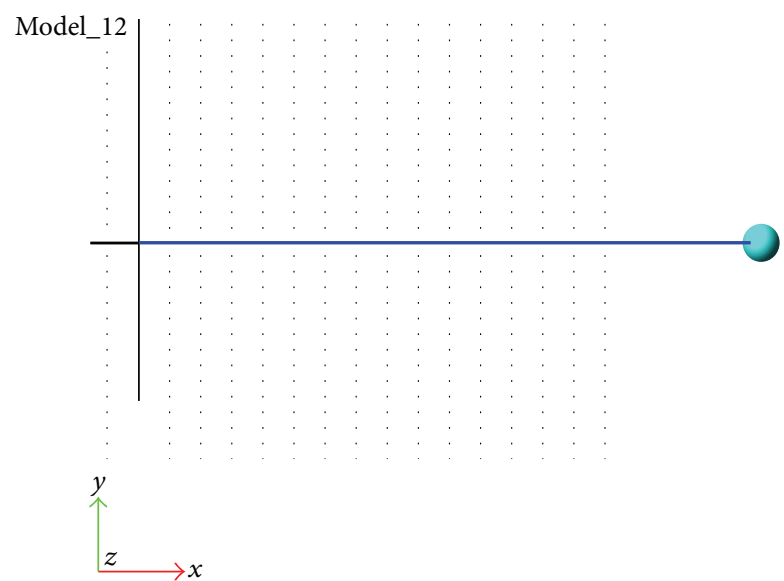

FIgURE 3: A fixed flexible arm in ADAMS

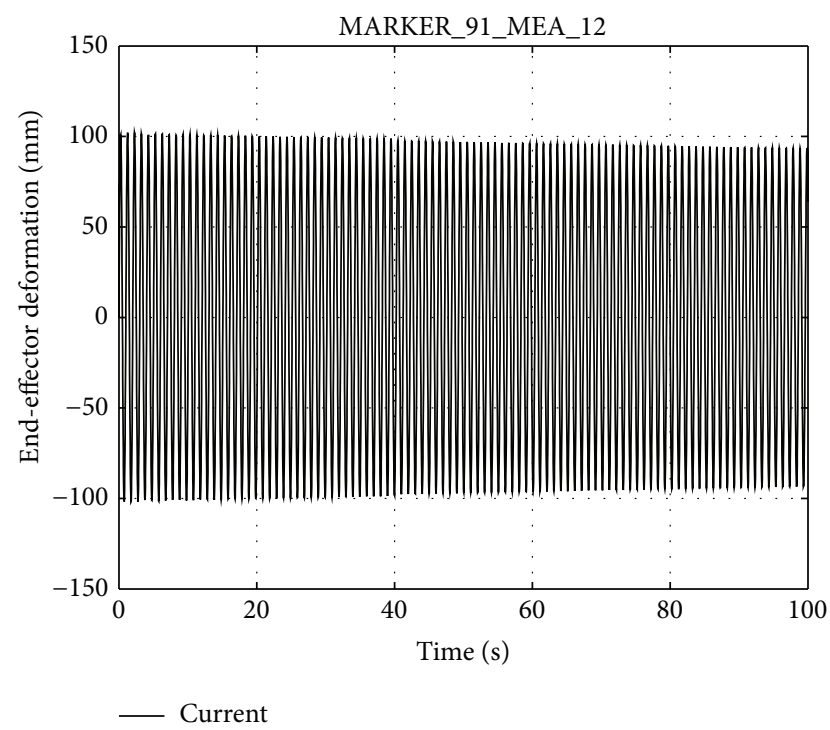

FIGURE 4: Response of the uncontrolled fixed flexible arm in ADAMS

In order to reduce vibration of the flexible structure via modal interaction, a vibration absorber is perpendicularly attached to the flexible arm at $x_{1}=0.5 \mathrm{~m}$, at which mass $m=0.3 \mathrm{~kg}$ and equilibrium position $s_{a}=0.05 \mathrm{~m}$, as shown in Figure 1. At the state of internal resonance, (24)(28) are integrated numerically in the absence of damping. The relationship between the modal amplitudes $a_{1}$ (dashed line) and $a_{2}$ (solid line) is shown in Figure 5. As can be seen, the peaks and troughs of the responses are exactly $180^{\circ}$ out of phase, verifying the fact that there is continuous exchange of the energy between modes of vibration.

When the damping of the vibration absorber is taken into account and $\zeta_{22}=0.0015,(24)-(28)$ are integrated numerically at the state of internal resonance. The motion of $a_{1}$ (dashed line) and $a_{2}$ (solid line) within the whole work process is illustrated in Figure 6. And the transfer of energy between modes can be found, verified by the coincidence of the peaks of one modal curve with the troughs of the other.

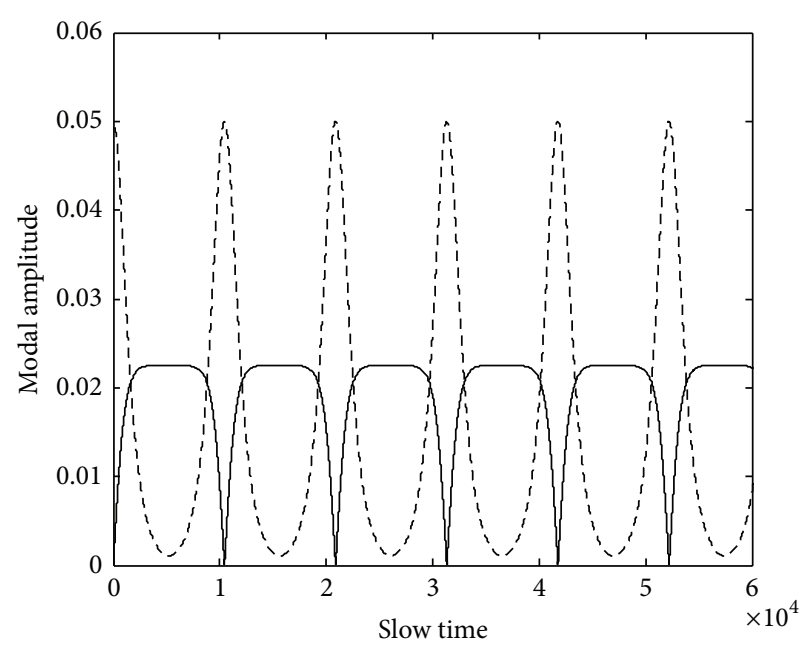

FIgURE 5: Undamped modal amplitudes in fixed flexible arm.

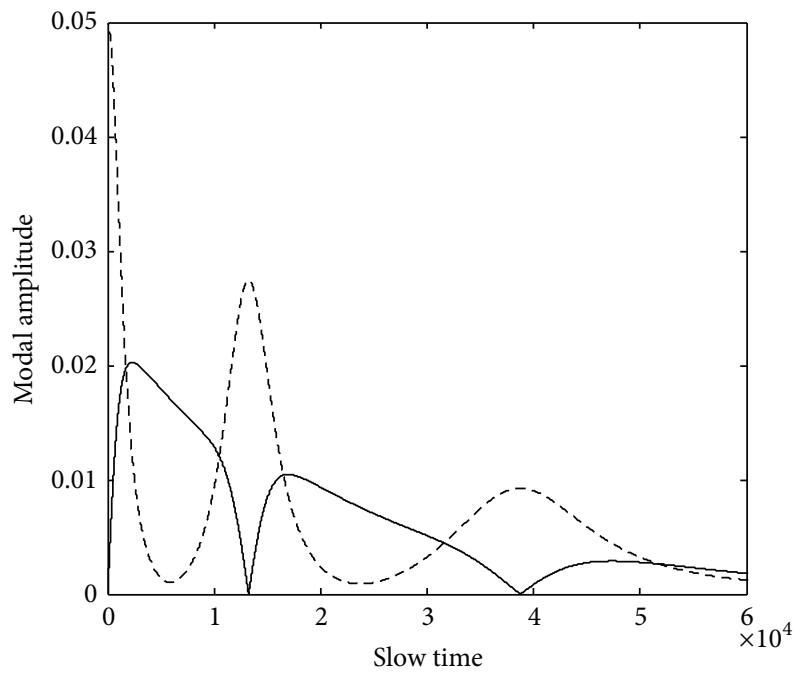

Figure 6: Damped modal amplitudes in fixed flexible arm.

In addition, the modal amplitudes decrease with time, which suggests that vibration energy of the fundamental mode of the flexible arm is absorbed by the absorber and dissipated through the damping of the absorber.

The end-effector deformation of the flexible arm equipped with the vibration absorber is shown in Figure 7. At the stage of 1:2 internal resonance, the initial deformation is reduced quickly within 10 seconds. After 20 seconds, the end-effector deformation is reduced by $50 \%$. After 40 seconds, the end-effector deformation is reduced by $80 \%$.

Through above simulation and analysis, it is verified that this control method based on internal resonance is effective in controlling vibration of the flexible structure.

Next, an example of the flexible arm undergoing rigid motion is considered. Suppose the desired joint motion of the arm is

$$
\dot{q}=0.2 \times \sin \left(\frac{\pi t}{3}\right)+0.5 \times \cos (t), \quad(0 \leq t \leq 100 \mathrm{~s}) .
$$




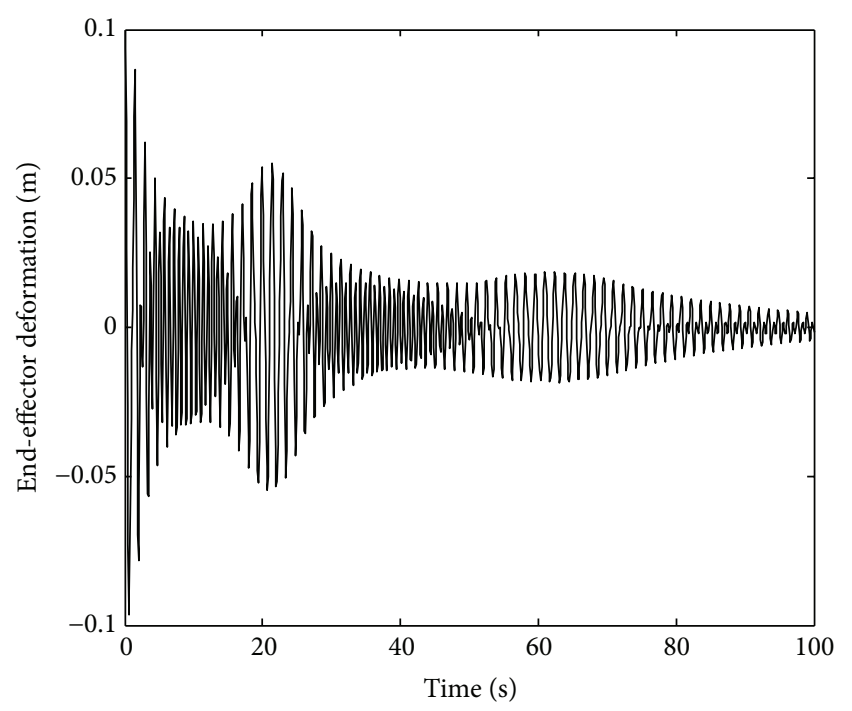

FIGURE 7: Response of the controlled fixed flexible arm.

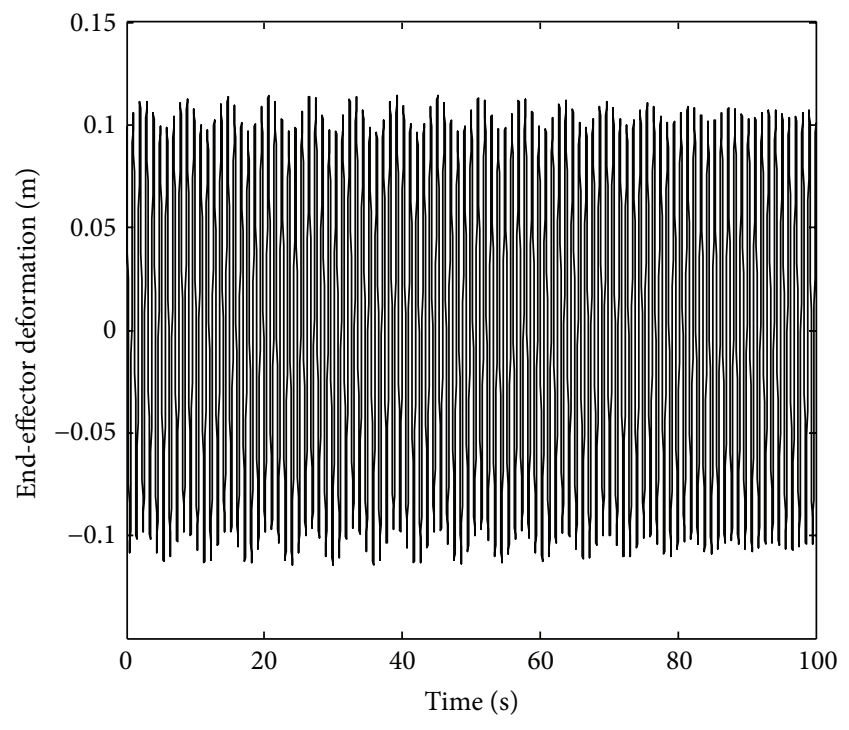

FIGURE 8: Response of the uncontrolled moving flexible arm.

If the flexible arm is not equipped with the vibration absorber, when moving according to (39), its end-effector deformation is obtained for the initial disturbance of $\delta(l, 0)=$ $0.1 \mathrm{~m}$, as shown in Figure 8. Compared with a flexible structure (as shown in Figure 2), rigid motion can cause more complex dynamic behaviors due to its coupling effect with flexural deformation. As can be seen, the end-effector response is no longer an attenuated vibration and its peaks even exceed the initial disturbance. Once again, the endeffector deformation is calculated using ADAMS software and shown in Figure 9. As can be seen, the results are similar to each other.

In order to reduce vibration of the flexible arm via modal interaction, a vibration absorber is perpendicularly attached to the flexible arm and the same parameters as in the aforementioned case are used. At the state of internal resonance,

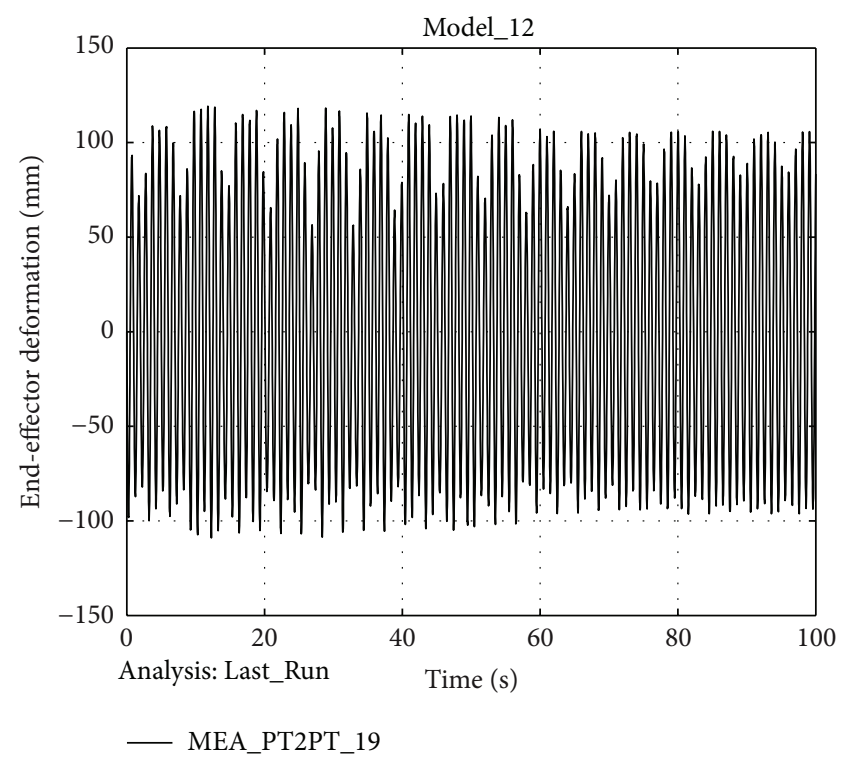

FIGURE 9: Response of the uncontrolled moving flexible arm in ADAMS.

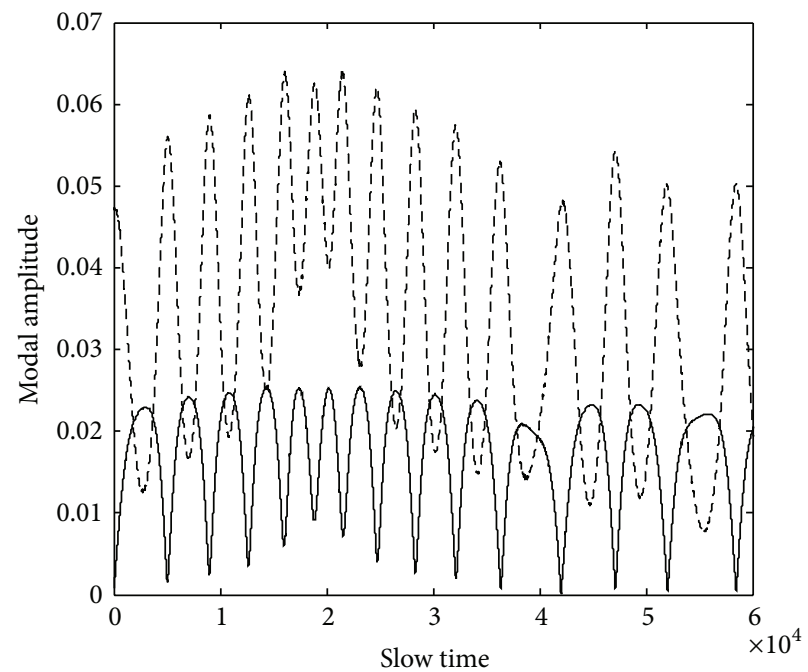

FIGURE 10: Undamped modal amplitudes in moving flexible arm.

(24)-(28) are integrated numerically in the absence of damping. The relationship between the modal amplitudes $a_{1}$ (dashed line) and $a_{2}$ (solid line) is shown in Figure 10. It can be seen that the peaks and troughs of the responses are exactly $180^{\circ}$ out of phase, which means that there is continuous exchange of the energy between modes of vibration.

When the damping of the vibration absorber is taken into account and $\zeta_{22}=0.005,(24)-(28)$ are integrated numerically at the state of internal resonance. The modal amplitudes $a_{1}$ (dashed line) and $a_{2}$ (solid line) are illustrated in Figure 11. Since each of $h_{2}$ and $h_{5}$ has relation to both $\zeta_{11}$ and $\zeta_{22}$, once $\zeta_{22}$ is added to the system, it can increase the damping of not only $a_{2}$ but also $a_{1}$. In this case, the vibration energy of $a_{1}$ can not only be absorbed by the damping of $a_{2}$ when transferred to $a_{2}$, but also be dissipated by its increased damping. 


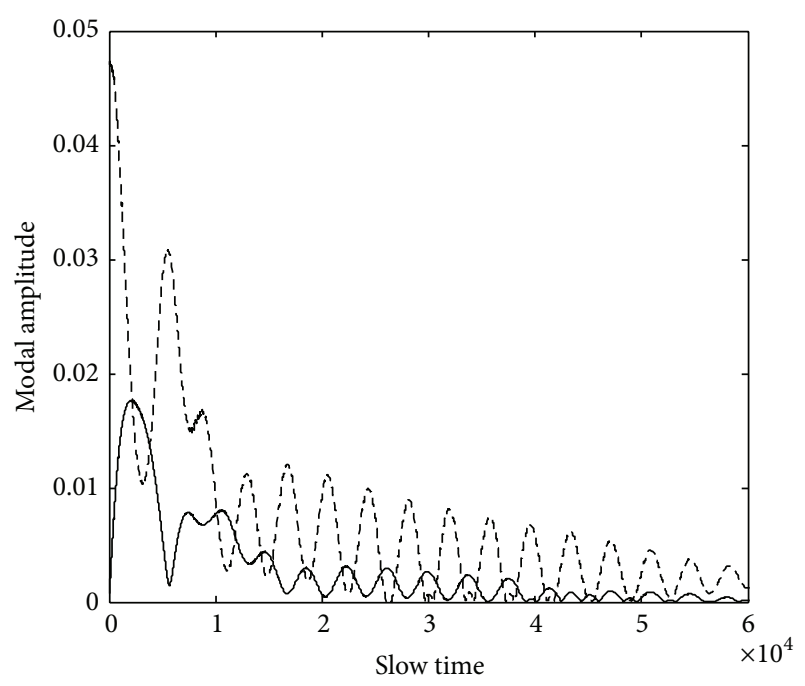

FIgURE 11: Damped modal amplitudes in moving flexible arm.

As demonstrated in Figure 11, the transfer of energy between modes can be found, verified by the coincidence of the peaks of one modal curve with the troughs of the other. In addition, the modal amplitudes decrease with time and approach the equilibrium value $\left(a_{1}=a_{2}=0\right)$, which suggests that the vibration energy of the flexible arm has been effectively absorbed and dissipated through internal resonance.

The end-effector deformation of the flexible arm equipped with the vibration absorber is shown in Figure 12. At the stage of 1:2 internal resonance, the end-effector deformation of the flexible arm is reduced quickly within 20 seconds. After 20 seconds, the end-effector deformation is reduced by $75 \%$, compared with the uncontrolled case (as shown in Figure 8). Final deformation of the end-effector can be reduced by $90 \%$. Also, an example of the initial disturbance of $\delta(l, 0)=0.2 \mathrm{~m}$ and $\zeta_{22}=0.005$ is conducted. The end-effector deformation is shown in Figure 13. As can be seen, even though larger disturbance is imposed on the system, vibration response can still be reduced effectively.

In addition, the effects of the absorber damping $\zeta_{22}$ are studied, as shown in Figure 14. It can be seen that if the absorber damping is very small, vibration energy flowing into the absorber cannot be effectively dissipated. However, if the absorber damping is too large, its effect becomes indistinctive. Therefore, an appropriate damping is necessary.

Furthermore, the effect of the absorber location is studied. When the absorber is attached to the flexible arm at $r=$ $0.35 \mathrm{~m}$, the controlled frequency of the arm is $1.0106 \mathrm{~Hz}$. Its end-effector deformation is shown in Figure 15. When the absorber is attached to the flexible arm at $r=0.5 \mathrm{~m}$, the controlled frequency of the arm is $0.9905 \mathrm{~Hz}$. Its end-effector deformation is shown in Figure 12. When the absorber is attached to the flexible arm at $r=0.75 \mathrm{~m}$, the controlled frequency of the arm is $0.9237 \mathrm{~Hz}$. Its end-effector deformation is shown in Figure 16. From these figures, it can be seen that the absorber location can remarkably affect the endeffector deformation, and an appropriate absorber location is necessary.

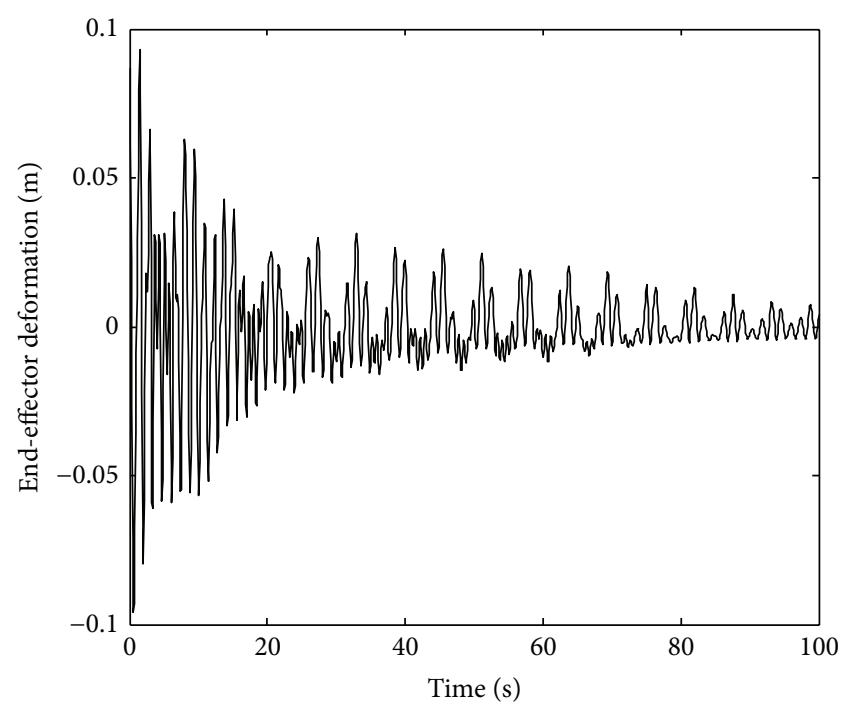

FIGURE 12: Response of the controlled $\operatorname{arm}(\delta(l, 0)=0.1 \mathrm{~m})$.

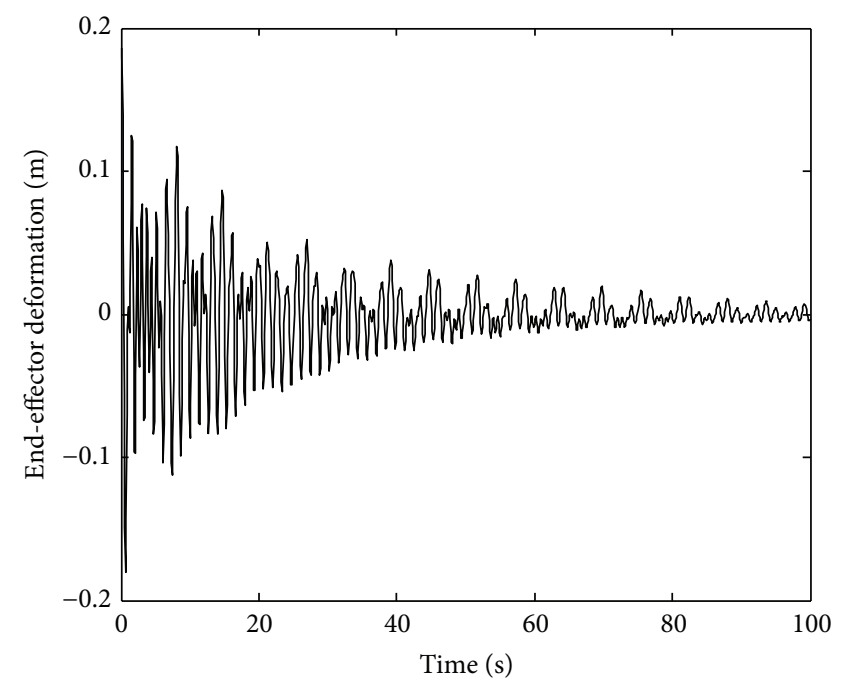

Figure 13: Response of the controlled arm $(\delta(l, 0)=0.2 \mathrm{~m})$.

Based on above simulations and analyses, it is proven that this method is effective in controlling nonlinear vibration of the flexible arm.

\section{Conclusion}

In this paper, a vibration control method based on energy migration is proposed to decrease vibration response of the flexible arm undergoing rigid motion. A type of vibration absorber is suggested and gives rise to the inertial coupling between the modes of the flexible arm and the absorber. By analyzing 1:2 internal resonance, it is proved that the internal resonance can be successfully created and the exchange of vibration energy is existent. Furthermore, due to the inertial coupling, the damping enhancement effect is revealed. Via the inertial coupling, vibration energy of the flexible arm can be dissipated by not only the damping of 

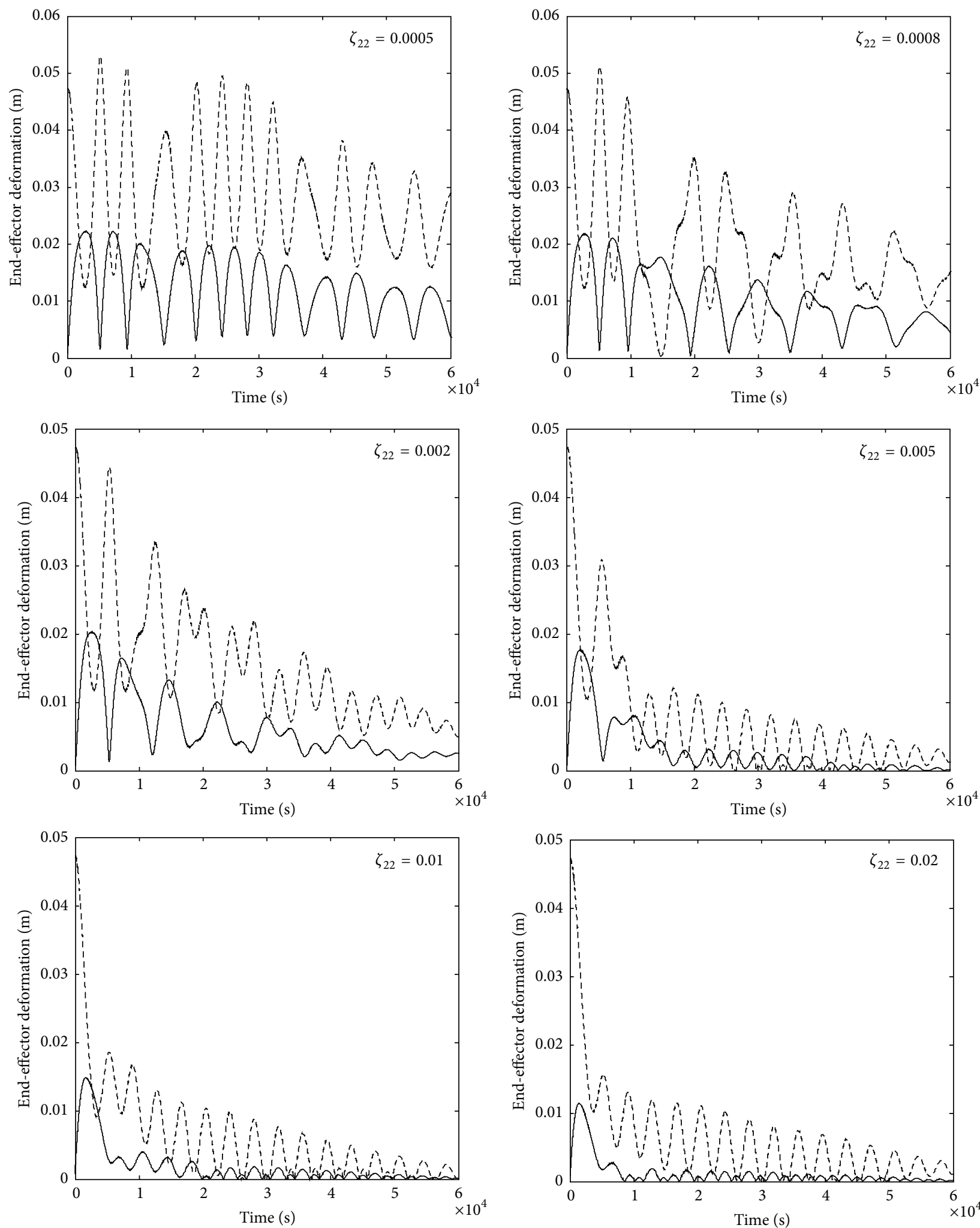

FIGURE 14: Effects of the absorber damping. 


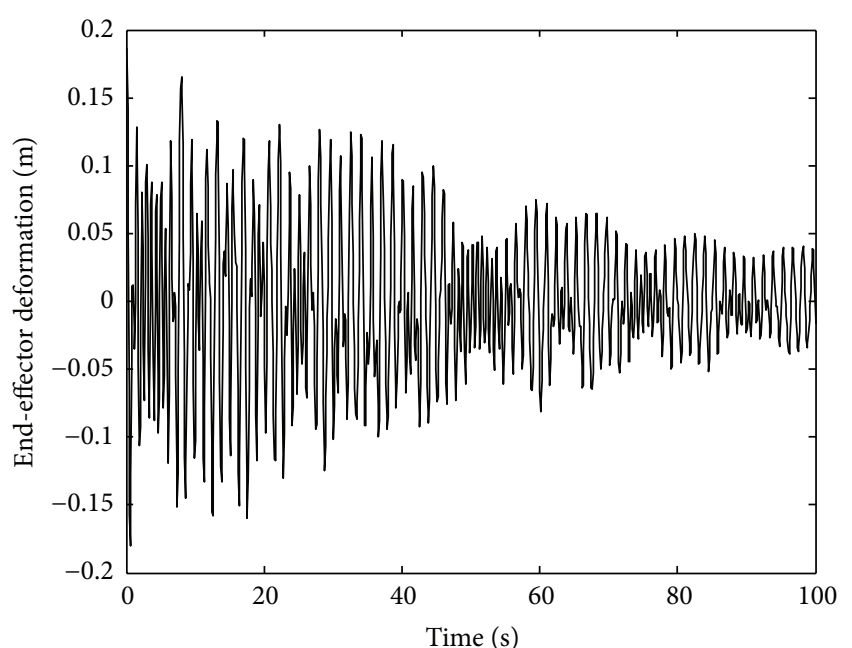

FIGURE 15: Response of the controlled arm $(r=0.35 \mathrm{~m})$.

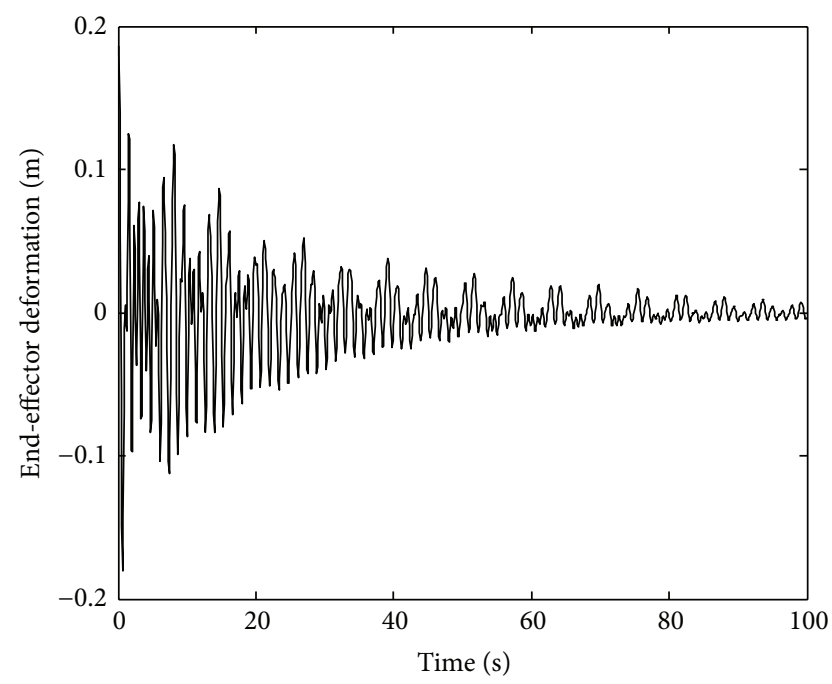

FIGURE 16: Response of the controlled arm $(r=0.75 \mathrm{~m})$.

the vibration absorber but also its own enhanced damping, thereby effectively decreasing vibration. Through numerical simulations and analyses, it is proven that this method is feasible in controlling nonlinear vibration of the flexible arm undergoing rigid motion.

\section{Conflict of Interests}

The authors declare that there is no conflict of interests regarding the publication of this paper.

\section{Acknowledgments}

This study is supported by the Pre-Research Foundation of GEH PLA (no. 9140A34030315KG18002) and the Major State Basic Research Development Program of China (973 Program) (no. 2013CB733000).

\section{References}

[1] L. L. Cui and Z. Q. Xiao, "Optimum structure design of flexible manipulators based on GA," in Proceedings of the IEEE International Conference on Intelligent Transportation Systems, vol. 2, pp. 1622-1626, October 2003.

[2] U. S. Dixit, R. Kumar, and S. K. Dwivedy, "Shape optimization of flexible robotic manipulators," Transactions of the ASMEJournal of Mechanical Design, vol. 128, no. 3, pp. 559-565, 2006.

[3] A. Ghazavi, F. Gordaninejad, and N. G. Chalhoub, "Dynamic analysis of a composite-material flexible robot arm," Computers \& Structures, vol. 49, no. 2, pp. 315-327, 1993.

[4] F. Gordaninejad, N. G. Chalhoub, A. Ghazavi, and Q. Lin, "Nonlinear deformation of a shear-deformable laminated compositematerial flexible robot arm," Journal of Mechanical Design, vol. 114, no. 1, pp. 96-102, 1992.

[5] S. K. Dwivedy and P. Eberhard, "Dynamic analysis of flexible manipulators, a literature review," Mechanism and Machine Theory, vol. 41, no. 7, pp. 749-777, 2006.

[6] M. Benosman and G. Le Vey, "Control of flexible manipulators: a survey," Robotica, vol. 22, no. 5, pp. 533-545, 2004.

[7] A. H. Nayfeh, Nonlinear Interactions, Wiley Series in Nonlinear Science, Wiley-Interscience, New York, NY, USA, 2000.

[8] G. Schmidt and A. Tondl, Non-Linear Vibrations, Cambridge University Press, New York, NY, USA, 1986.

[9] M. F. Golnaraghi, "Regulation of flexible structures via nonlinear coupling," Dynamics and Control, vol. 1, no. 4, pp. 405-428, 1991.

[10] K. L. Tuer, M. F. Golnaraghi, and D. Wang, "Development of a generalised active vibration suppression strategy for a cantilever beam using internal resonance," Nonlinear Dynamics, vol. 5, no. 2, pp. 131-151, 1994.

[11] S. S. Oueini and M. F. Golnaraghi, "Experimental implementation of the internal resonance control strategy," Journal of Sound and Vibration, vol. 191, no. 3, pp. 377-396, 1996.

[12] A. Khajepour, M. F. Golnaraghi, and K. A. Morris, "Application of center manifold theory to regulation of a flexible beam," Journal of Vibration and Acoustics, Transactions of the ASME, vol. 119, no. 2, pp. 158-164, 1997.

[13] P. F. Pai, B. Rommel, and M. J. Schulz, "Non-linear vibration absorbers using higher order internal resonances," Journal of Sound and Vibration, vol. 234, no. 5, pp. 799-817, 2000.

[14] O. N. Ashour and A. H. Nayfeh, "Adaptive control of flexible structures using a nonlinear vibration absorber," Nonlinear Dynamics, vol. 28, no. 3-4, pp. 309-322, 2002.

[15] M. Yaman and S. Sen, "Determining the effect of detuning parameters on the absorption region for a coupled nonlinear system of varying orientation," Journal of Sound and Vibration, vol. 300, no. 1-2, pp. 330-344, 2007.

[16] C. K. Hui, Y. Y. Lee, and C. F. Ng, "Use of internally resonant energy transfer from the symmetrical to anti-symmetrical modes of a curved beam isolator for enhancing the isolation performance and reducing the source mass translation vibration: theory and experiment," Mechanical Systems and Signal Processing, vol. 25, no. 4, pp. 1248-1259, 2011. 

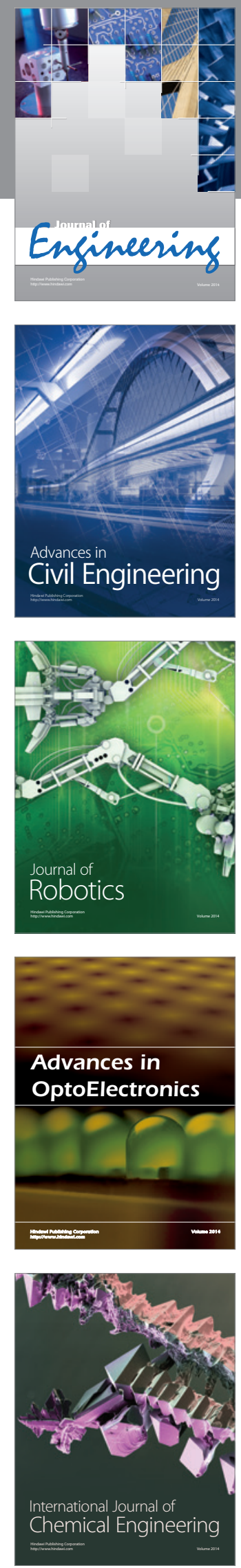

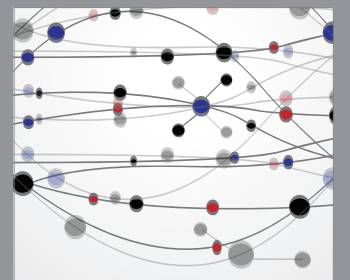

The Scientific World Journal
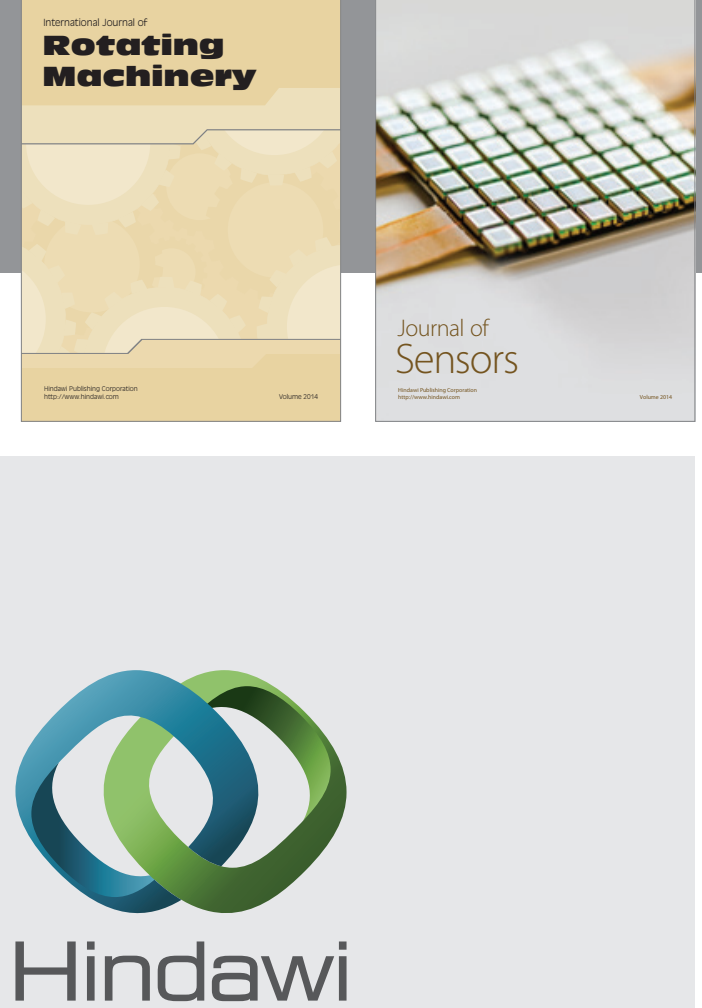

Submit your manuscripts at http://www.hindawi.com
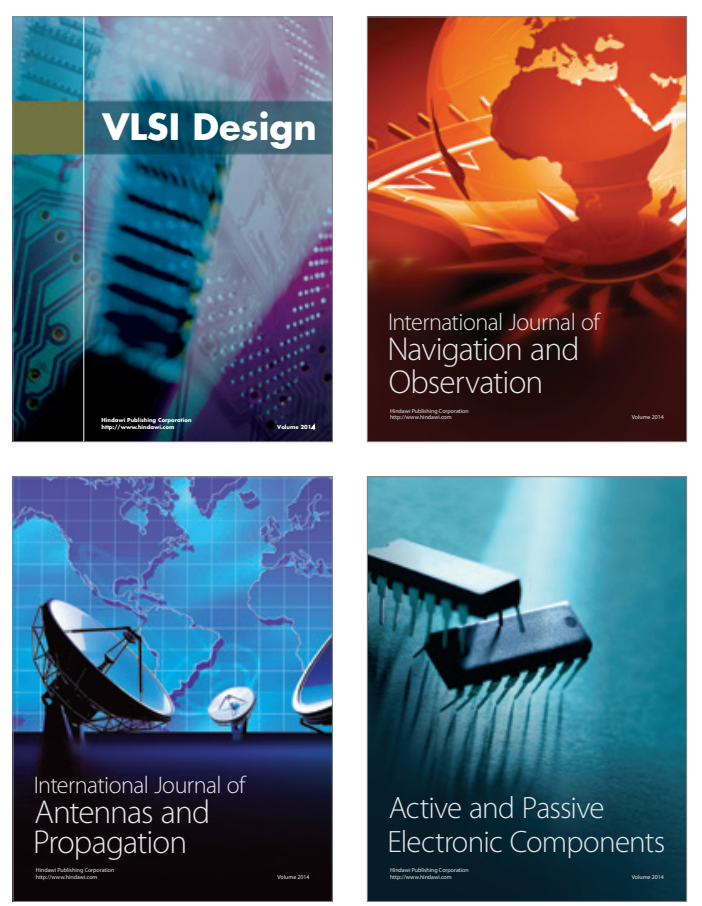
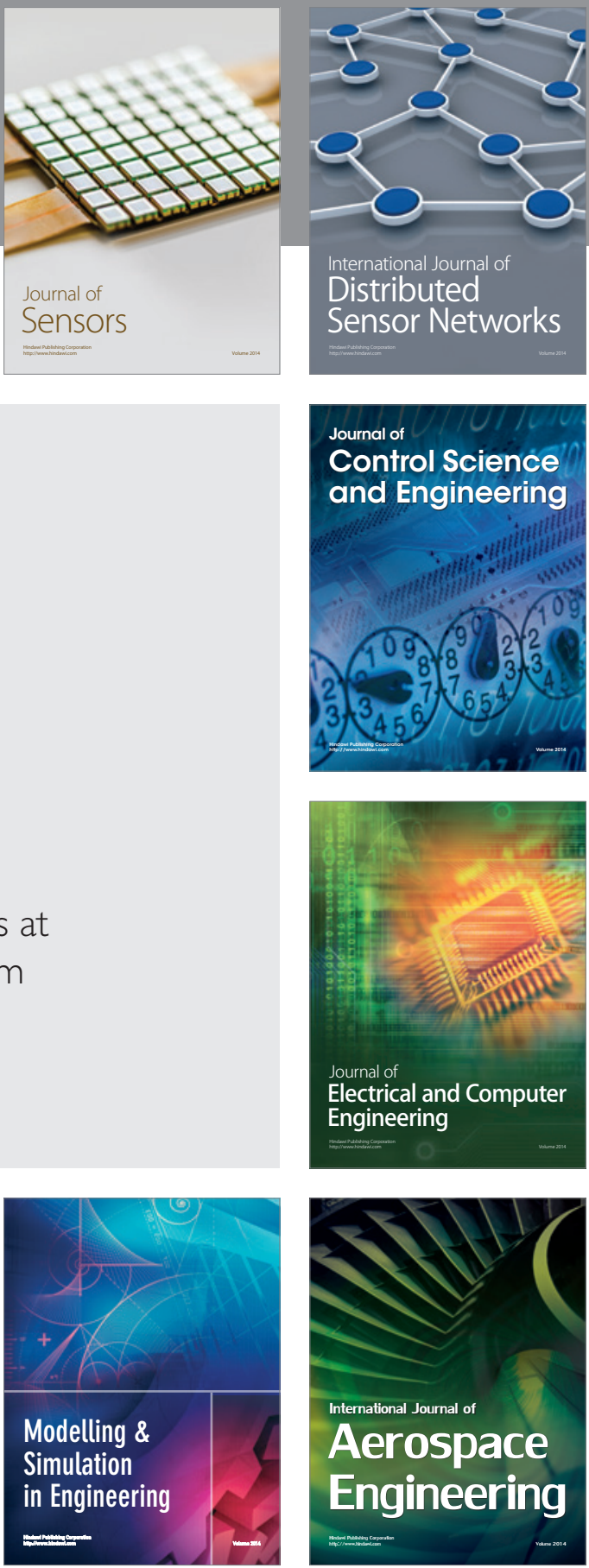

Journal of

Control Science

and Engineering
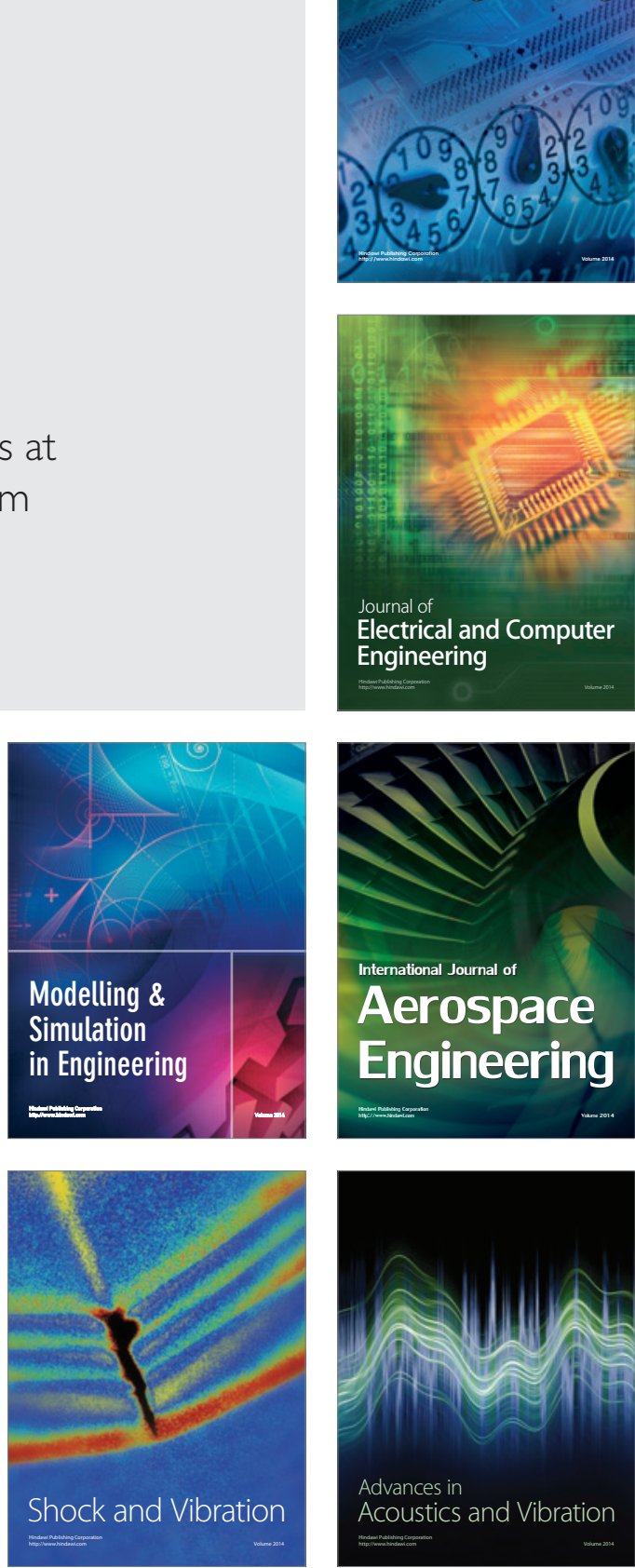